\title{
Exosomes from breast cancer cells can convert adipose tissue- derived mesenchymal stem cells into myofibroblast-like cells
}

\author{
JUNG AH CHO*, HO PARK*, EUN HYE LIM and KYO WON LEE \\ Adult Stem Cell Research Institute, Department of Obstetrics and Gynecology, Kangbuk \\ Samsung Hospital, Sungkyunkwan University School of Medicine, Seoul, Republic of Korea
}

Received June 22, 2011; Accepted August 11, 2011

DOI: $10.3892 /$ ijo.2011.1193

\begin{abstract}
Exosomes are small membrane vesicles secreted into the extracellular environment by various types of cells, including tumor cells. Exosomes are enriched with a discrete set of cellular proteins, and therefore expected to exert diverse biological functions according to cell origin. Mesenchymal stem cells (MSCs) possess the potential for differentiation into multilineages and can also function as precursors for tumor stroma including myofibroblast that provides a favorable environment for tumor progression. Although a close relationship between tumor cells and MSCs in a neoplastic tumor microenvironment has already been revealed, how this communication works is poorly understood. In this study, we investigated the influence of tumor cell-derived exosomes on MSCs by treating adipose tissuederived MSCs (ADSCs) with breast cancer-derived exosomes. The exosome-treated ADSCs exhibited the phenotypes of tumor-associated myofibroblasts with increased expression of $\alpha$-SMA. Exosome treatment also induced increased expression of tumor-promoting factors SDF-1, VEGF, CCL5 and TGF $\beta$. This phenomenon was correlated with increased expression of TGF $\beta$ receptor I and II. Analysis of SMAD2, a key player in the TGF $\beta$ receptor-mediated SMAD pathway, revealed that its phosphorylation was increased by exosome treatment and was inhibited by treatment with SB431542, an inhibitor of the SMAD-mediated pathway, resulting in decreased expression of $\alpha$-SMA. Taken together, our results show that tumor-derived exosomes induced the myofibroblastic phenotype and functionality in ADSCs via the SMAD-mediated signaling pathway. In
\end{abstract}

Correspondence to: Dr Kyo Won Lee, Adult Stem Cell Research Institute, Department of Obstetrics and Gynecology, Kangbuk Samsung Hospital, Sungkyunkwan University School of Medicine, 108 PyungDong, Jongro-Gu, Seoul 110-746, Republic of Korea

E-mail: kw4773.lee@samsung.com

\section{${ }^{*}$ Contributed equally}

Abbreviations: MSCs, mesenchymal stem cells, ADSCs, adiposederived mesenchymal stem cells

Key words: tumor-derived exosomes, mesenchymal stem cells, adipose tissue-derived stem cells, tumor microenvironment, tumor stroma, myofibroblast conclusion, this study suggests that tumor-derived exosomes can contribute to progression and malignancy of tumor cells by converting MSCs within tumor stroma into tumor-associated myofibroblasts in the tumor microenvironment.

\section{Introduction}

Most tumor tissue is composed of two principal structures (1): the parenchyma, or tumor cells, and the stroma, a supportive tissue including connective tissue and vessels. Many studies have shown that all tumors have and require stroma for nutritional support and for the removal of waste products (2-5). Tumor-associated stroma are commonly observed components in all neoplastic tissues and play a fundamental role in tumor growth, invasion, and dissemination by interacting with carcinoma cells. Accordingly, tumor growth and metastasis can be controlled by tumor-associated stromal cells (2-5). One type of tumor-associated stromal cell, myofibroblasts, develops in the neoplastic environment as tumor cells grow and progress $(6,7)$. Tumor-associated myofibroblasts play an essential role in tumor cell metastasis by stimulating angiogenesis via secretion of SDF-1 and by controlling the formation of the extracellular matrix via secretion of matrix metalloprotease $(6,7)$.

A potential origin of stromal cells is mesenchymal stem cells (MSCs). MSCs are multipotent adult stem cells that can selfrenew and have the capability of differentiating into a variety of cell types including osteoblasts, chondrocytes, myocytes, and adipocytes, as well as several different types of stromal cells including muscle, cartilage, marrow stroma, and fibroblasts $(8,9)$. Although MSCs were initially found in the marrow stroma, they have also been obtained from several different tissues including umbilical cord blood, amniotic fluid, and adipose tissue as well as bone marrow (10-13).

In addition to their properties of self renewal and their multilineage potential, MSCs have been reported to have another unique characteristic of 'tropism migration', which can migrate to damaged tissue sites such as sites with inflammation or wounds, and sites with cancer cells $(14,15)$. There is accumulating evidence that MSCs are potential precursors for tumor stroma and could be targeted delivery vehicles for anticancer agents $(16,17)$. An important study published by Karnoub et al demonstrated that MSCs within the tumor stroma promoted breast tumor metastasis (18), clearly suggesting an interaction between MSCs and cancer cells in tumor progression and 
malignancy. In addition, MSCs may be associated with tumor propagation or dissemination by preventing recognition of the tumor cells from the immune system as well as promoting tumor cell invasiveness $(19,20)$. Therefore, prior reports have indicated a close relationship between MSCs and cancer cells.

Molecules secreted by tumor cells might be key players in such interactions. A study by McAllister et al revealed that a molecule secreted by breast cancer cells instigated indolent tumor outgrowth by inducing activation and mobilization of stromal precursor cells (21). Another study has shown that a cancer-derived molecule stimulated MSCs to differentiate into myofibroblast-like cells (22). In this regard, exosomes are good candidates for this activity.

Exosomes are small membrane vesicles (40-90 $\mathrm{nm}$ in diameter) originating from one of intracellular compartments, called multivesicular bodies (MVBs), that release their internal vesicles into the extracellular space by direct fusion with plasma membranes (23). These vesicles are produced by various cell types including reticulocytes, intestinal epithelial cells, and hematopoietic cells, as well as tumor cells (24). Proteomic analyses have determined that exosomes contain a selective enrichment of a discrete set of cellular proteins associated with antigen presentation, signal transduction, and migration/adhesion including major histocompatibility complex (MHC) molecules, heat shock proteins (HSPs), MFG-E8, and tetraspanins $(25,26)$. In addition, exosomes also contain cellular RNAs such as mRNAs and microRNAs, which can be delivered to other cells and can be functional in new locations (27).

A variety of tumor cells constitutively release exosomes, and exosomes are present in large amounts in different types of cancer patient as well as cancer cell supernatants (28). Nevertheless, the function of these tumor-derived exosomes is not well defined yet. Since tumor-derived exosomes were revealed to contain tumorspecific antigens and tumor peptide/MHC class I complexes that can be recognized by tumor-specific CTLs, a role as a cell-free antigen source for anticancer vaccines has been suggested (29). However, it has also been shown that tumor-derived exosomes can promote tumor growth rather than inhibit it, possibly due to negative effects of tumor-derived exosomes on IL-2-induced CTL responses via TGF- $\beta$, which enhances regulatory $\mathrm{T}$ cell induction (30,31). Moreover, accumulating evidence indicates that tumor-derived exosomes exert a broad array of detrimental effects on the immune system, ranging from apoptosis in activated anti-tumor $\mathrm{T}$ cells to impairment of monocyte differentiation into dendritic cells and induction of myeloid suppressive cells (32). In addition, tumor-derived exosomes are predicted to play a role in chemoresistance-related pharmacological modulation of tumor cells, and are considered potential players in intercellular communications (32).

In this study, we examined the potential role and related molecular mechanisms of tumor-derived exosomes in communication between tumor cells and MSCs in the neoplastic tumor microenvironment using exosomes from two different breast cancer cell lines and adipose tissue-derived MSCs (ADSCs), which are abundantly resident in breast tissue.

\section{Materials and methods}

Isolation and culture of mesenchymal stem cells from adipose tissue (ADSCs). Adipose tissue obtained from lipoaspirates was washed with sterile phosphate-buffered saline (PBS) to remove contaminating debris and red blood cells $(33,34)$. The washed aspirates were treated with $0.075 \%$ collagenase (type I; SigmaAldrich, St. Louis, MO, USA) in PBS for $60 \mathrm{~min}$ at $37^{\circ} \mathrm{C}$ with gentle agitation, followed by inactivation with an equal volume of DMEM/10\% fetal bovine serum (FBS). After centrifugation for $10 \mathrm{~min}$ at low speed, the cellular pellet was resuspended in DMEM $/ 10 \%$ FBS and filtered through a $100-\mu \mathrm{m}$ mesh filter to remove debris. The filtrate was centrifuged as before and plated onto conventional tissue culture plates in control medium (DMEM, 10\% FBS, 1\% antibiotic/antimycotic solution) and maintained at $37^{\circ} \mathrm{C}$ in a $\mathrm{CO}_{2}$ incubator. The ADSCs were used after 3-5 passages throughout this study. Each donor provided fully informed consent at the Kangbuk Samsung Hospital. The study protocols have been approved by the institutional review board (IRB) of Kangbuk Samsung Hospital.

Exosome isolation and purification. MCF-7 or MDA-MB231 breast adenocarcinoma cell lines were cultured in media supplemented with $10 \%$ FBS that was previously centrifuged at $100,000 \mathrm{x}$ g overnight to eliminate bovine-derived exosomes. The method used to isolate exosomes was described previously (35). Cell culture supernatant was collected to isolate exosomes by successive centrifugation $(300 \mathrm{x} \mathrm{g}$ for $5 \mathrm{~min}, 1,200 \mathrm{x} \mathrm{g}$ for $20 \mathrm{~min}$, and $10,000 \mathrm{x} \mathrm{g}$ for $30 \mathrm{~min}$ ) and then pelleted by ultracentrifugation at $100,000 \mathrm{x} g$ for $1 \mathrm{~h}$. Finally, the exosomal pellet was washed in a large volume of PBS and then resuspended in PBS. For further purification, exosomes were resuspended in $2.5 \mathrm{M}$ sucrose in $20 \mathrm{mM}$ Hepes buffer ( $\mathrm{pH} 7.4$ ) and subsequently loaded into the bottom of a SW41 tube. Hepes buffer $(20 \mathrm{mM})$ with $2 \mathrm{M}$ sucrose was carefully loaded on top of the exosomes followed by Hepes buffer $(20 \mathrm{mM})$ with $0.25 \mathrm{M}$ sucrose to produce a discontinuous 2-0.25 M sucrose gradient. After spinning overnight at 100,000 $\mathrm{x}$ g in an SW41 swing rotor, $1 \mathrm{ml}$ of each fraction was collected and centrifuged at $100,000 \mathrm{x}$ g for $1 \mathrm{~h}$. After aspirating the supernatant, the pellet was resuspended in PBS.

Exosome treatment and sample preparation. The quantity of exosomal protein was determined using the bicinchoninic acid (BCA) method (Pierce Biotechnology, Rockford, IL). Exosomes $(0,4$, or $20 \mu \mathrm{g})$ were added to ADSCs that had been plated in 6-well plates and adapted to serum-free medium. For inhibitor treatment, cells were treated with $10 \mu \mathrm{M}$ SB431542 (SigmaAldrich) for $30 \mathrm{~min}$ before the addition of exosomes. The treated cells were subjected to the following experiments.

Immunocytochemistry. ADSCs were grown on a Lab-tek chamber slide (177429, NUNC, Naperville, IL, USA) overnight. The exosome-treated ADSCs were fixed at room temperature (RT) for $10 \mathrm{~min}$, and then washed four times with filtered PBS, followed by blocking with $3 \%$ FBS in $1 \%$ bovine serum albumin (BSA)/PBS for 30 min at RT. After washing three times with filtered PBS, the cells were incubated overnight at $4^{\circ} \mathrm{C}$ with primary antibody against $\alpha$-SMA ( $\alpha$ smooth muscle actin, Dako, CA, USA) and subsequently incubated with Alexa 488-conjugated secondary antibody for $30 \mathrm{~min}$ at RT, followed by staining with $1 \mathrm{ml} 10 \mu \mathrm{g} / \mathrm{ml}$ DAPI solution (Molecular Probes, CA, USA) for $10 \mathrm{~min}$. After washing with PBS, images were obtained at 200x fold magnification using a fluorescence microscope (Carl Zeiss, Germany). 
A.

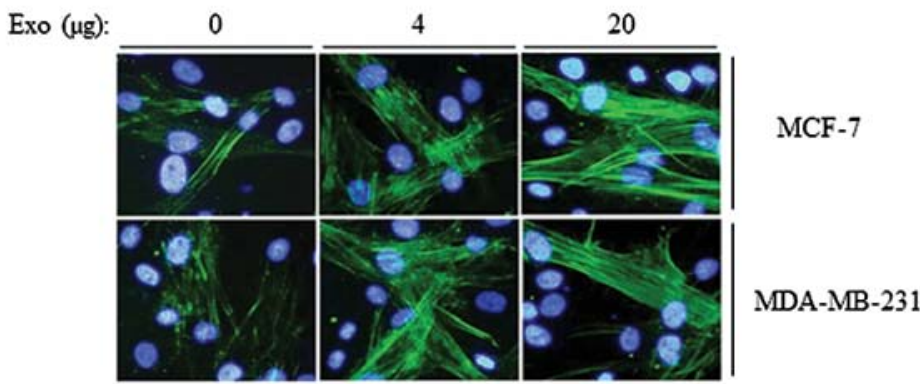

B.
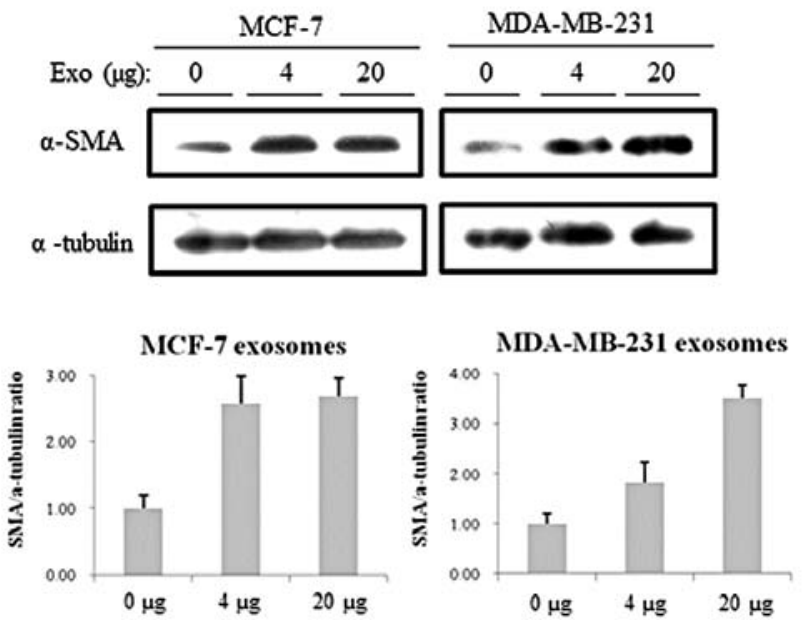

Figure 1. Analysis of the expression of a myofibroblastic marker in ADSCs treated with exosomes from MCF-7 and MDA-MB-231 cells. ADSCs were incubated with 0,4 or $20 \mu \mathrm{g}$ of exosomes isolated from two different breast adenocarcinoma cell lines, MCF7 and MDA-MB-231. (A) Immunocytochemistry was performed using the exosome-treated MSCs by incubating with a primary antibody against $\alpha$-SMA and Alexa 488-conjugated secondary antibody (green). The images were obtained at 200x magnification by fluorescence microscopy after counterstaining with the nuclear-staining dye DAPI (blue). (B) Total cell proteins were extracted from the exosome-treated ADSCs and assessed by Western blotting using primary antibodies against $\alpha$-SMA as a myofibroblast marker or $\alpha$-tubulin as a protein loading control, and horse radish peroxidase (HRP)-tagged secondary antibody. The image was obtained using an ECL chemiluminescence system, and the band intensity was analyzed using ImageJ software, shown as a graph on the bottom panel.

$R T$-PCR. Total RNA was extracted using the TRIzol method (Invitrogen, CA, USA). Briefly, cells were lysed in TRIzol, and subsequently mixed with chloroform. After centrifugation, the clear top phase was collected and mixed with isopropanol, followed by centrifugation. The pellet was washed with $75 \%$ ethanol solution and harvested by centrifugation as before. The final pellet was air-dried for $10 \mathrm{~min}$ and resuspended in DEPCtreated water. After quantification, $3 \mu \mathrm{g}$ of RNA was used for the synthesis of complementary DNA (cDNA) with $5 \mathrm{X}$ reaction buffer, RNasin Ribonuclease inhihitor (Promega, USA), $50 \mu \mathrm{M}$ oligo-dT (Invitrogen), $2.5 \mathrm{mM}$ dNTP (Takara, Japan), and M-MLV reverse transcriptase (Promega). One microgram of the cDNA was used as a template for subsequent PCR amplification using Taq polymerase premix [1.25 units $\mathrm{Taq}^{\mathrm{TM}}, 1.6 \mathrm{mM} \mathrm{dNTP}$, $20 \mathrm{mM}$ Tris- $\mathrm{HCl}$ (pH 8.3), $100 \mathrm{mM} \mathrm{KCl}$, and $3 \mathrm{mM} \mathrm{MgCl}{ }_{2}$, Takara] plus $20 \mathrm{pM}$ of each primer which were reacted in a Thermocycler (Biometra, Germany) for 35 cycles of $1 \mathrm{~min}$ at $90^{\circ} \mathrm{C}, 1 \mathrm{~min}$ at $55^{\circ} \mathrm{C}$, and $1 \mathrm{~min}$ at $72^{\circ} \mathrm{C}$. The PCR products were visualized in a $1.5 \%$ agarose-containing gel by staining with ethidium bromide (EtBr).

Western blot analysis. Cells were lysed in RIPA buffer $(50 \mathrm{mM}$ Tris- $\mathrm{HCl}, 150 \mathrm{mM} \mathrm{NaCl}, 1 \% \mathrm{NP}-40,0.25 \%$ Na-deoxycholate, $1 \mathrm{mM}$ EDTA, $1 \mathrm{mM}$ PMSF, $1 \mu \mathrm{g} / \mathrm{ml}$ aprotinin/leupeptin/ pepstain, $1 \mathrm{mM} \mathrm{Na} \mathrm{VO}_{4}, 1 \mathrm{mM} \mathrm{NaF}$ ) for $10 \mathrm{~min}$ on ice and then centrifuged at $10,000 \mathrm{x} g$ at $4^{\circ} \mathrm{C}$ to remove cell debris. The protein in the supernatant was quantified using the BCA method (Pierce), resolved by SDS-PAGE, and transferred to a PVDF membrane (Millipore, Billerica, MA, USA) that was pretreated with methanol. The membrane was blocked with 5\% BSA in TTBS (10 mM Tris, $150 \mathrm{mM} \mathrm{NaCl,} \mathrm{0.1 \%} \mathrm{Tween} \mathrm{20)} \mathrm{for} 1 \mathrm{~h}$ at RT and subsequently incubated with primary antibodies overnight at $4^{\circ} \mathrm{C}$. After vigorous washing in TTBS, the blots were incubated with horseradish peroxidase (HRP)-tagged secondary antibodies for $30 \mathrm{~min}$. The labeled proteins of interest were visualized by exposing the blots to X-ray film in a dark room using an enhanced chemiluminescence (ECL) kit (Amersham Biosciences, Buckinghamshire, England).

\section{Results}

Tumor-derived exosomes can induce expression of a myofbroblast marker in MSCs. To examine whether tumor-derived exosomes play a role in the generation of tumor-associated myofibroblasts from MSCs, we treated adipose tissue-derived MSCs (ADSCs) with exosomes from two different breast adenocarcinoma cell lines, MCF-7 and MDA-MB-231. First, we investigated whether the tumor-derived exosomes could 
A.

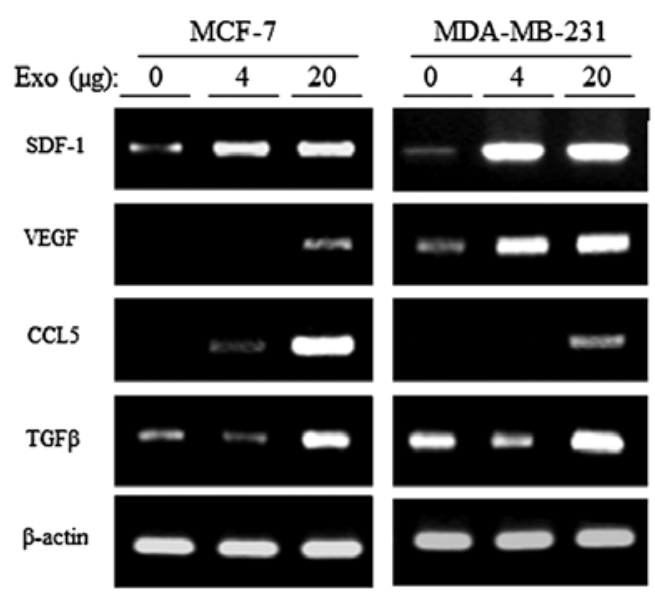

B.
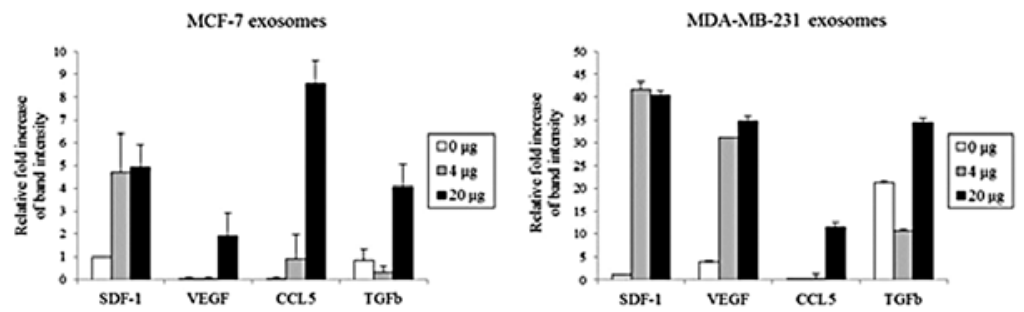

Figure 2. Analysis of several tumor-promoting factors in ADSCs treated with exosomes from MCF-7 and MDA-MB-231 cells. ADSCs were incubated with 0, 4 or $20 \mu \mathrm{g}$ of exosomes isolated from two different breast adenocarcinoma cell lines, MCF7 and MDA-MB-231. Total RNA was extracted from the exosome-treated ADSCs and analyzed by RT-PCR with specific primer pairs for SDF-1, CCL5, VEGF and TGF $\beta$, which promote angiogenesis and metastasis of tumors. $\beta$-actin was used as an internal control. The PCR products were separated by electrophoresis and visualized using a gel documentation system with EtBr staining (A), and the band intensity was analyzed using ImageJ software, shown as a graph (B).

A.

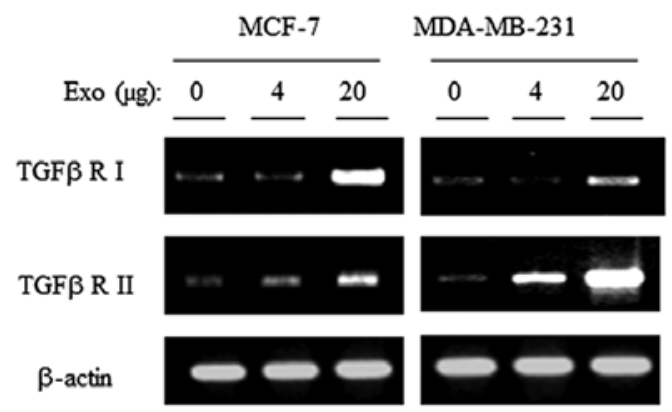

B.
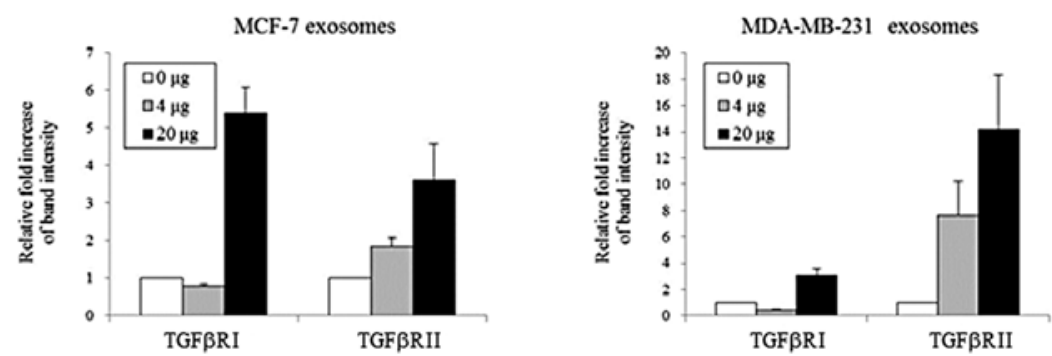

Figure 3. Analysis of surface receptor expression in ADSCs treated with exosomes from MCF-7 and MDA-MB-231 cells. ADSCs were incubated with 0,4, or $20 \mu \mathrm{g}$ of exosomes isolated from two different breast adenocarcinoma cell lines, MCF7 and MDA-MB-231. Total RNA was extracted from the exosome-treated ADSCs and analyzed by RT-PCR with specific primer pairs for two isoforms of the TGF $\beta$ receptor, TGF $\beta$ receptor I (TGF $\beta$ R I) and II (TGF $\beta$ R II). $\beta$-actin was used as an internal control. The PCR products were separated by electrophoresis and visualized using a gel documentation system with EtBr staining (A). The band intensity was analyzed using ImageJ software, shown as a graph (B). 


\section{A. SMAD pathway}

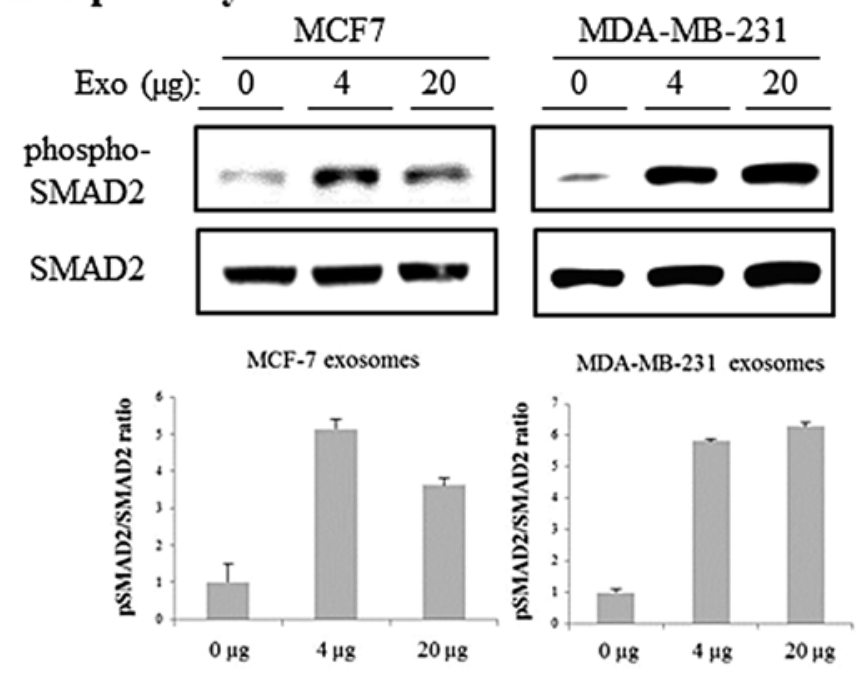

\section{B. ERK pathway}
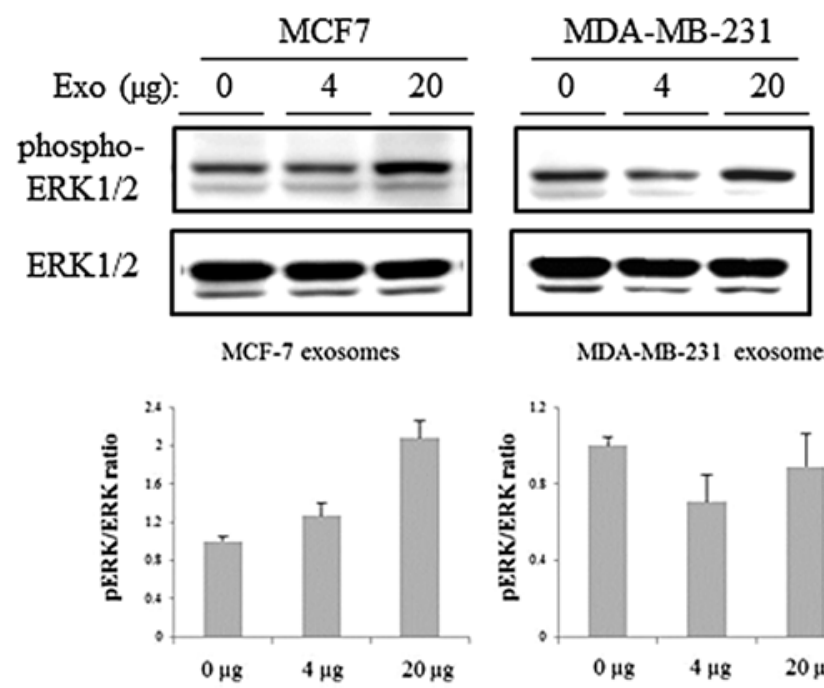

MDA-MB-231 exosomes

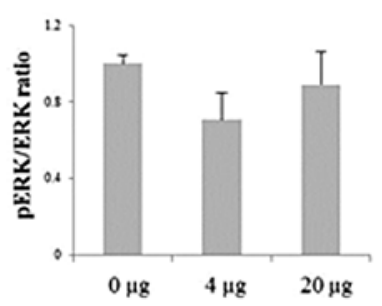

Figure 4. Analysis of signaling pathways activated in ADSCs by treatment with exosomes from MCF-7 and MDA-MB-231 cells. ADSCs were incubated with 0, 4 , or $20 \mu \mathrm{g}$ of exosomes isolated from two different breast adenocarcinoma cell lines, MCF7 and MDA-MB-231. Total cell proteins were extracted from the exosometreated ADSCs and assessed by Western blotting using antibodies against SMAD2 and the phosphorylated form of SMAD2 (phospho-SMAD2) for the SMAD pathway (A), or ERK and the phosphorylated form of ERK (phospho-ERK) for the ERK pathway (B). After subsequent incubation with HRP-tagged secondary antibodies, the image was obtained using the ECL chemiluminescence system, and the band intensity was analyzed using ImageJ software, shown as a graph on the bottom panel.

induce the MSCs to have a phenotypic characteristic of tumorassociated myofibroblasts. As tumor-associated myofibroblasts express $\alpha$-smooth muscle actin ( $\alpha$-SMA) (36), we performed immunocytochemistry and Western blot analysis using ADSCs that were treated with exosomes from the two breast cancer cell lines (Fig. 1). As shown in Fig. 1A, the results of immunocytochemical staining against $\alpha$-SMA demonstrated that $\alpha$-SMA expression was markedly elevated in the ADSCs by treatment with both types of exosomes. Consistent with this finding, Western blot analysis showed that the expression of $\alpha$-SMA protein was noticeably increased in a dose-dependent manner by treatment with both exosomes (Fig. 1B). These results demonstrated that the breast cancer-derived exosomes induced expression of a myofibroblastic marker in the ADSCs, suggesting that tumor-derived exosomes can cause MSCs to have a myofibroblastic phenotype.

Tumor-derived exosomes can induce the production of myofibroblast-associated functional factors in MSCs. As tumorassociated myofibroblasts can promote tumor malignancy by secretion of tumor-promoting factors (6,7), we next investigated whether ADSCs with the myofibroblastic phenotype can produce functional factors that support and promote tumor growth. As shown in Fig. 2, the results of RT-PCR revealed that the expression of several tumor-supportive cytokines such as SDF-1, VEGF, CCL5, and TGF $\beta$ was induced or greatly increased in the ADSCs by both exosome treatments, indicating that tumor-derived exosomes can induce the production of myofibroblast-associated 


\section{A. pSMAD2}

\begin{tabular}{|c|c|c|c|c|c|c|c|c|c|c|c|c|}
\hline \multirow[b]{2}{*}{$\operatorname{Exo}(\mu \mathrm{g}):$} & \multicolumn{6}{|c|}{ MCF-7 } & \multicolumn{6}{|c|}{ MDA-MB-231 } \\
\hline & 0 & 4 & 20 & 0 & 4 & 20 & 0 & 4 & 20 & 0 & 4 & 20 \\
\hline SB431542 $(10 \mu \mathrm{M})$ & - & - & - & + & + & + & - & - & - & + & + & + \\
\hline pSMAD2 & $=$ & 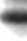 & & & & tent & - & - & 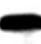 & - & & $\longrightarrow$ \\
\hline SMAD2 & & & & & & & & & $\ldots$ & - & & - \\
\hline
\end{tabular}

B. $\alpha$-SMA

\begin{tabular}{|c|c|c|c|c|c|c|c|c|c|c|c|c|}
\hline \multirow{2}{*}{ Exo $(\mu \mathrm{g})$} & \multicolumn{6}{|c|}{ MCF-7 } & \multicolumn{6}{|c|}{ MDA-MB-231 } \\
\hline & 0 & 4 & 20 & 0 & 4 & 20 & 0 & 4 & 20 & 0 & 4 & 20 \\
\hline SB431542 (10 $\mu \mathrm{M}):$ & - & - & - & + & + & + & - & - & - & + & + & + \\
\hline$\alpha$-SMA & $=$ & - & $=$ & - & - & - & - & & & - & & $\infty$ \\
\hline$\alpha$-tubulin & & & & & & & & & & & & \\
\hline
\end{tabular}

Figure 5. Confirmation of the signaling pathway activated in ADSCs by treatment with exosomes from MCF-7 and MDA-MB-231 cells. ADSCs were incubated with 0 , 4, or $20 \mu \mathrm{g}$ of exosomes from MCF-7 and MDA-MB-231 cells. For inhibitor treatment, cells were incubated with $10 \mu \mathrm{M} \mathrm{SB} 431542$ for 30 min before the addition of exosomes. Total cell proteins were extracted from the treated ADSCs and assessed by Western blotting using antibodies against SMAD2 and the phosphorylated form of SMAD2 (pSMAD2) (A), or $\alpha$-SMA and $\alpha$-tubulin (B). After subsequent incubation with HRP-tagged secondary antibodies, the image was obtained using the ECL chemiluminescence system.

functional cytokines from MSCs. Together with the above results, these data suggest that tumor exosomes can induce MSCs to acquire the physical and functional characteristics of tumor-supporting myofibroblasts.

Tumor-derived exosomes activate the TGF $\beta$ receptor-mediated signaling pathway in MSCs to have the physical and functional characteristics of myofibroblasts . Next, we investigated which molecules were involved in the acquisition of myofibroblastic characteristics of the ADSCs. Since exosomes must pass through the cell surface to enter the cell, we predicted that a surface receptor would be activated during this process. As a primary candidate, we investigated the expression of TGF $\beta$ receptors because tumor-derived exosomes are known to function in immune suppression via TGF $\beta(30,31)$. The results shown in Fig. 3 demonstrated that the expression of two TGF $\beta$ receptor isotypes, TGF $\beta$ R I and II, was prominently increased by the exosome treatment, showing the same pattern as the previously presented data in Figs. 1 and 2. These results indicated that exosomes from tumor cells might cause MSCs to have the physical and functional characteristics of myofibroblasts possibly through activation of TGF $\beta$ receptor-mediated signaling pathways in the cells.

Tumor-derived exosomes can stimulate the SMAD2-mediated signaling pathway in MSCs with physical and functional characteristics of myofibroblasts. To define the molecular mechanism involved in the differentiation process of ADSCs to myofibroblasts, we evaluated two representative signaling pathways among the downstream pathways of TGF $\beta$ receptormediated signaling, which are either SMAD-dependent or SMAD-independent pathways. To evaluate the activation levels of these pathways following exosome treatment, we performed Western blot analysis against the phosphorylated form of SMAD2 for the SMAD-dependent pathway or the phosphorylated form of ERK for the ERK pathway, a representative SMAD-independent pathway. As shown in Fig. 4, exosomes from MCF-7 and MDA-MB-231 cells both induced an increase in the phosphorylated form of SMAD2 (Fig. 4A), while exosomes from MCF-7 cells simultaneously induced an increase in the phosphorylated form of ERK (Fig. 4B). This result demonstrated that the SMAD2-mediated SMADdependent pathway was principally activated in the ADSCs by the exosome treatments.

To confirm the activation of SMAD2 by the tumor-derived exosomes, we treated the ADSCs with SB431542, a specific inhibitor of the SMAD-dependent pathway. As shown in Fig. 5, the increase in the phosphorylated form of SMAD2 induced by the tumor-derived exosomes was clearly blocked by inhibitor treatment (Fig. 5A), and consistent with this result, the increase in $\alpha$-SMA expression induced by the tumor-derived exosomes was also inhibited by SB431542 (Fig. 5B). This result demonstrated that SMAD2 activation was involved in the increased expression of $\alpha$-SMA induced in the ADSCs by the breast cancer-derived exosomes, suggesting that the ADSCs acquired the characteristics of myofibroblasts by exosomes from breast cancer cells principally via activation of the SMAD-dependent signaling pathway. Collectively, these data indicate that tumorderived exosomes can convert MSCs into tumor-associated myofibroblasts by activating a SMAD2-mediated intracellular signaling pathway.

\section{Discussion}

Most cells, including tumor cells, release exosomes that harbor a discrete set of proteins from their originating cells, implying the potential of unique activities and functions (24). A growing body of evidence provides clues about the functions of exosomes 
from tumor cells, which can play an active role in several biological behaviors of tumor cells including immune modulation, pharmacological modulation, and their relationship with the host environment (32). Although tumor-derived exosomes can be used as a powerful anti-cancer vaccines through genetic or non-genetic modulation to increase their anti-tumor effects $(37,38)$, it is more likely that tumor-derived exosomes possess an in vivo function that reflects the tumor cell properties because they are revealed to accumulate in the body fluids of cancer patients. Considering that tumor-derived exosomes may exert a range of different effects at once because they contain not a single macromolecule (protein or gene) but a complex of such macromolecules, much remains to be understood about the biological functions of tumor-derived exosomes.

Mesenchymal stem cells (MSCs) exhibit 'tropism migration' toward wounds, injury, and inflammation that results from various paracrine and endocrine signals. MSCs can also migrate to tumor sites that are considered to be 'never-healing wounds' and compose tumor stroma (39). Because of such properties, MSCs are considered to be potential efficient vehicles for cancer therapeutic genes (40). The tumor stroma plays a supporting role in the propagation or dissemination of tumors by producing pro-tumorigenic cytokines (41). A prior study demonstrated that exposure to tumor cell-conditioned medium promoted migration of MSCs with an increase in production of stromal cell-derived factor (SDF)-1 protein (42). More importantly, a study was carried out showing that MSCs within the tumor stroma promote breast cancer metastasis by CCL5 signaling through the chemokine receptor CCR5 expressed in the tumors (18). Our previous studies demonstrated that cytokines secreted by MSCs can influence tumor cell growth $(43,44)$. Therefore, manipulation of the interaction between MSCs within the tumor stroma and tumor cells might improve conventional cancer therapy. The results presented in this study reveal the effects of tumor-derived exosomes in the interaction with MSCs in the tumor microenvironment where stromal cells and cancer cells communicate.

Myofibroblasts, or tumor-associated fibroblasts, are key components of tumor stroma because they secrete various paracrine factors that support rapid tumor growth and metastasis, and thereby contribute to tumor aggressiveness and poor prognostic outcomes (45). Although it is known that these cells can be specifically detected by their $\alpha$-SMA expression (36), there are few studies on how the myofibroblasts differentiate and develop in the tumor stroma. As shown in Fig. 1, treatment of ADSCs with exosomes from both of the breast cancer cell lines resulted in increased expression of $\alpha$-SMA, implying that myofibroblastic cells can be generated from MSCs within the tumor stroma in the tumor microenvironment in response to tumor-derived exosomes.

In addition, the tumor-derived exosomes caused induction or increased expression of the tumor-promoting factors SDF-1, VEGF1, CCL5, and TGF $\beta$ in the ADSCs that exhibited $\alpha$-SMA expression, as shown in Fig. 2. SDF-1 and VEGF are the representative angiogenic factors for neo-vascularization (46), and CCL5 is the main player in the role of MSCs in breast cancer metastasis, as mentioned above (18). TGF $\beta$ is known to promote tumor cell growth via its immunosuppressive role, which can induce a defective signaling cascade in immune cells including $\mathrm{T}$ or NK cells and the genera- tion of myeloid suppressor cells or regulatory T cells (32). Accordingly, we can predict that tumor-derived exosomes are involved in angiogenesis and metastasis of tumor cells, as well as the immune cell suppression that contributes to tumor cell progression and malignancy.

Consistent with the results of a previous study demonstrating the effect of a tumor-derived factor in the differentiation of MSCs to myofibroblast-like cells (22), our study shows that exosomes secreted from tumor cells are candidates for the differentiation process possibly through activation of TGF $\beta$ receptor-mediated signalng pathway. Because tumor-derived exosomes are known to contain functional TGF $\beta(30,31)$, we considered a possibility for changes in expression of TGF $\beta$ receptors of ADSCs by exosomes. As expected, exosome treatment resulted in increased expression of both TGF $\beta$ and TGF $\beta$ receptors (Figs. 2 and 3). Although it is not yet clear whether the increased expression of TGF $\beta$ receptors was due to the exosomal TGF $\beta$ (31) or to the autocrine effect of TGF $\beta$ generated in the ADSCs by the exosome treatment, as in Fig. 3, the results of our study show that expression of the TGF $\beta$ receptors was increased and that the subsequent downstream SMAD2-mediated signaling pathway was activated in the ADSCs by exosomes from two different breast cancer cell lines (Fig. 4). All of the results obtained in this study clearly indicate that tumor-derived exosomes can induce the myofibroblastic phenotype and functionality in MSCs by activating the SMAD-mediated signaling pathway as a principal mechanism, which implies the importance of tumor-derived exosomes in interactions between tumor cells and adjacent tissue-resident stem cells. Furthermore, we assume that, although MSCs can be attracted to tumor sites via tropism migration as mentioned above, their property of promoting tumor cell metastasis might be acquired within the tumor microenvironment by interaction with tumorderived molecules including exosomes.

As shown in Fig. 4, the exosomes from the two breast cancer cell lines showed different effects in the activation of intracellular signaling pathways; exosomes from MCF-7 cells, but not MDA-MB-231 cells, activated the ERK-mediated signaling pathway as well as the SMAD-mediated pathway. This result implies that exosomes can stimulate other signaling pathways in addition to the SMAD-mediated pathway. We hypothesize that this effect is due to the unique contents that each type of exosomes may differently harbor since their originating cells, MCF-7 and MDA-MB-231, have their own characteristics with respect to various biological behaviors, including genetic and physiological aspects $(47,48)$. Accordingly, exosomes from different tumor cells may specifically exert their own biological effects in addition to common effects according to their context.

Human diseases caused by tumors cannot be fully cured without destruction of the tumor stroma, which may support relapse of tumor cells if present (49). In this study, we identified a new potential role of tumor-derived exosomes in the interaction of tumor cells with MSCs in the tumor microenvironment, demonstrating that tumor-derived exosomes may contribute to the progression and malignancy of tumor cells by the biogenesis of tumor-associated myofibroblastic cells from MSCs in tumor stroma. The results of this study demonstrate for the first time the importance of tumor- 
derived exosomes in the generation of tumor stroma, providing a foundation for new target discovery focusing on blockade or inhibition of molecules or activities related to tumor-derived exosomes, such as exosome biogenesis, secretion, or uptake, as a novel promising approach for cancer therapy.

\section{Acknowledgements}

This study was supported by a grant of the National R\&D Program for Cancer Control, Ministry for Health, Welfare and Family Affairs, Republic of Korea (0820250). We have no financial conflicts.

\section{References}

1. Dvorak HF, Nagy JA and Dvorak AM: Structure of solid tumors and their vasculature: implications for therapy with monoclonal antibodies. Cancer Cells 3: 77-85, 1991.

2. Dickson DJ and Shami PJ: Angiogenesis in acute and chronic leukemias. Leuk Lymphoma 42: 847-853, 2001.

3. Nagy JA, Meyers MS, Masse EM, Herzberg KT and Dvorak HF: Pathogenesis of ascites tumor growth: fibrinogen influx and fibrin accumulation in tissues lining the peritoneal cavity. Cancer Res 55: 369-375, 1995.

4. Senger DR, Galli SJ, Dvorak AM, Perruzzi CA, Harvey VS and Dvorak HF: Tumor cells secrete a vascular permeability factor that promotes accumulation of ascites fluid. Science 219: 983-985, 1983.

5. Folkman J and Shing Y: Angiogenesis. J Biol Chem 267: 10931-10934, 1992.

6. Orimo A and Weinberg RA: Stromal fibroblasts in cancer: a novel tumor-promoting cell type. Cell Cycle 5: 1597-1601, 2006.

7. Desmouliere A, Guyot C and Gabbiani G: The stroma reaction myofibroblast: a key player in the control of tumor cell behavior. Int J Dev Biol 48: 509-517, 2004.

8. Chamberlain G, Fox J, Ashton B and Middleton J: Concise review: mesenchymal stem cells: their phenotype, differentiation capacity, immunological features, and potential for homing. Stem Cells 25: 2739-2749, 2007.

9. Caplan AI and Bruder SP: Mesenchymal stem cells: building blocks for molecular medicine in the 21st century. Trends Mol Med 7: 259-264, 2001.

10. Schaffler A and Buchler C: Concise review: adipose tissue-derived stromal cells-basic and clinical implications for novel cell-based therapies. Stem Cells 25: 818-827, 2007.

11. In 't Anker PS, Scherjon SA, Kleijburg-van der Keur C, et al: Isolation of mesenchymal stem cells of fetal or maternal origin from human placenta. Stem Cells 22: 1338-1345, 2004.

12. Prockop DJ, Sekiya I and Colter DC: Isolation and characterization of rapidly self-renewing stem cells from cultures of human marrow stromal cells. Cytotherapy 3: 393-396, 2001.

13. Lu LL, Liu YJ, Yang SG, et al: Isolation and characterization of human umbilical cord mesenchymal stem cells with hematopoiesis-supportive function and other potentials. Haematologica 91: 1017-1026, 2006.

14. Sasaki M, Abe R, Fujita Y, Ando S, Inokuma D and Shimizu H: Mesenchymal stem cells are recruited into wounded skin and contribute to wound repair by transdifferentiation into multiple skin cell type. J Immunol 180: 2581-2587, 2008.

15. Spaeth E, Klopp A, Dembinski J, Andreeff M and Marini F: Inflammation and tumor microenvironments: defining the migratory itinerary of mesenchymal stem cells. Gene Ther 15: 730-738, 2008.

16. Hung SC, Deng WP, Yang WK, et al: Mesenchymal stem cell targeting of microscopic tumors and tumor stroma development monitored by noninvasive in vivo positron emission tomography imaging. Clin Cancer Res 11: 7749-7756, 2005.

17. Franz M, Richter P, Geyer C, et al: Mesenchymal cells contribute to the synthesis and deposition of the laminin-5 gamma2 chain in the invasive front of oral squamous cell carcinoma. J Mol Histol 38 : 183-190, 2007

18. Karnoub AE, Dash AB, Vo AP, et al: Mesenchymal stem cells within tumour stroma promote breast cancer metastasis. Nature 449: 557-563, 2007.
19. Ohno S, Tachibana M, Fujii T, Ueda S, Kubota H and Nagasue N: Role of stromal collagen in immunomodulation and prognosis of advanced gastric carcinoma. Int J Cancer 97: 770-774, 2002.

20. Keleg S, Buchler P, Ludwig R, Buchler MW and Friess H: Invasion and metastasis in pancreatic cancer. Mol Cancer 2: 14, 2003.

21. McAllister SS, Gifford AM, Greiner AL, et al: Systemic endocrine instigation of indolent tumor growth requires osteopontin. Cell 133: 994-1005, 2008

22. Jeon ES, Moon HJ, Lee MJ, et al: Cancer-derived lysophosphatidic acid stimulates differentiation of human mesenchymal stem cells to myofibroblast-like cells. Stem Cells 26: 789-797, 2008.

23. Denzer K, Kleijmeer MJ, Heijnen HF, Stoorvogel W and Geuze HJ: Exosome: from internal vesicle of the multivesicular body to intercellular signaling device. J Cell Sci 113 Pt 19: 3365-3374, 2000.

24. Keller S, Sanderson MP, Stoeck A and Altevogt P: Exosomes: from biogenesis and secretion to biological function. Immunol Lett 107: 102-108, 2006

25. Mathivanan S, Ji H and Simpson RJ: Exosomes: extracellular organelles important in intercellular communication. J Proteomics 73: 1907-1920, 2010.

26. Van Niel G, Porto-Carreiro I, Simoes S and Raposo G: Exosomes: a common pathway for a specialized function. J Biochem 140: 13-21, 2006.

27. Valadi H,Ekstrom K, Bossios A, Sjostrand M,Lee JJ and Lotvall JO: Exosome-mediated transfer of mRNAs and microRNAs is a novel mechanism of genetic exchange between cells. Nat Cell Biol 9: 654-659, 2007.

28. Andre F, Schartz NE, Chaput N, et al: Tumor-derived exosomes: a new source of tumor rejection antigens. Vaccine 20 (Suppl. 4): S28-S31, 2002

29. Wang DG, Sun SZ, Wang ZG and Wang X: [Study of induction of tumor specific cytotoxic T lymphocyte by using tumor-derived exosome]. Hua Xi Kou Qiang Yi Xue Za Zhi 24: 160-163, 2006.

30. Clayton A, Mitchell JP, Court J, Mason MD and Tabi Z: Human tumor-derived exosomes selectively impair lymphocyte responses to interleukin-2. Cancer Res 67: 7458-7466, 2007.

31. Xiang X, Poliakov A, Liu C, et al: Induction of myeloid-derived suppressor cells by tumor exosomes. Int J Cancer 124: 2621-2633, 2009.

32. Iero M, Valenti R, Huber V, et al: Tumour-released exosomes and their implications in cancer immunity. Cell Death Differ 15: 80-88, 2008.

33. Raposio E, Guida C, Baldelli I, et al: Characterization and induction of human pre-adipocytes. Toxicol In Vitro 21: 330-334, 2007.

34. Zuk PA, Zhu M, Mizuno H, et al: Multilineage cells from human adipose tissue: implications for cell-based therapies. Tissue Eng 7: 211-228, 2001

35. Raposo G, Nijman HW, Stoorvogel W, et al: B lymphocytes secrete antigen-presenting vesicles. J Exp Med 183: 1161-1172, 1996.

36. Kinner B, Zaleskas JM and Spector M: Regulation of smooth muscle actin expression and contraction in adult human mesenchymal stem cells. Exp Cell Res 278: 72-83, 2002.

37. Cho JA, Lee YS, Kim SH, Ko JK and Kim CW: MHC independent anti-tumor immune responses induced by Hsp70-enriched exosomes generate tumor regression in murine models. Cancer Lett 275: 256-265, 2009.

38. Cho JA, Yeo DJ, Son HY, et al: Exosomes: a new delivery system for tumor antigens in cancer immunotherapy. Int J Cancer 114: 613-622, 2005.

39. Studeny M, Marini FC, Dembinski JL, et al: Mesenchymal stem cells: potential precursors for tumor stroma and targeted-delivery vehicles for anticancer agents. J Natl Cancer Inst 96: 1593-1603, 2004.

40. Kucerova L, Altanerova V, Matuskova M, Tyciakova S and Altaner C: Adipose tissue-derived human mesenchymal stem cells mediated prodrug cancer gene therapy. Cancer Res 67: 6304-6313, 2007.

41. Rowley DR: What might a stromal response mean to prostate cancer progression? Cancer Metastasis Rev 17: 411-419, 1998.

42. Menon LG, Picinich S, Koneru R, et al: Differential gene expression associated with migration of mesenchymal stem cells to conditioned medium from tumor cells or bone marrow cells. Stem Cells 25: 520-528, 2007.

43. Cho JA, Park H, Kim HK, et al: Hyperthermia-treated mesenchymal stem cells exert antitumor effects on human carcinoma cell line. Cancer 115: 311-323, 2009. 
44. Park H, Cho JA, Kim SK, Kim JH and Lee SH: Hyperthermia on mesenchymal stem cells (MSCs) can sensitize tumor cells to undergo cell death. Int J Hyperthermia 24: 638-648, 2008.

45. Ruiter D, Bogenrieder T, Elder D and Herlyn M: Melanomastroma interactions: structural and functional aspects. Lancet Oncol 3: 35-43, 2002.

46. Yu JX, Huang XF, Lv WM, et al: Combination of stromal-derived factor-lalpha and vascular endothelial growth factor gene-modified endothelial progenitor cells is more effective for ischemic neovascularization. J Vasc Surg 50: 608-616, 2009.
47. Ross DT and Perou CM: A comparison of gene expression signatures from breast tumors and breast tissue derived cell lines. Dis Markers 17: 99-109, 2001.

48. Lacroix M and Leclercq G: Relevance of breast cancer cell lines as models for breast tumours: an update. Breast Cancer Res Treat 83: 249-289, 2004

49. Vincent $\mathrm{T}$ and Mechti $\mathrm{N}$ : Extracellular matrix in bone marrow can mediate drug resistance in myeloma. Leuk Lymphoma 46: 803-811, 2005. 